\title{
Evolución y desarrollo de sistema interactivo para ayudar a infantes con Trastorno de Espectro Autista en su integración a escuelas convencionales
}

\section{Evolution and development of an interactive system to help infants with Autism Spectrum Disorder in their integration into conventional schools}

\author{
ORTIZ-SIMÓN, José Luis †** AGUILERA-HERNÁNDEZ, Martha, MURILLO-RODRÍGUEZ, \\ Miguel Randall y OLIVARES-CABALLERO, Daniel
}

Tecnológico Nacional de México / Instituto Tecnológico de Nuevo Laredo, Mexico.

ID $1^{\mathrm{er}}$ Autor: José Luis, Ortiz-Simón / ORC ID: 0000-0001-6548-3849, CVU CONACYT ID: 209883

ID $1^{\mathrm{er}}$ Coautor: Martha, Aguilera-Hernández / ORC ID: 0000-0001-8127-190X, CVU CONACYT ID: 19115

ID $2^{\text {do }}$ Coautor: Miguel Randall, Murillo-Rodríguez / ORC ID: 0000-0002-9906-2230

ID $3^{\text {er }}$ Coautor: Daniel, Olivares-Caballero

\begin{abstract}
Resumen
Las capacidades cognitivas de niños con Trastorno de Espectro Autista (TEA) son diferentes a las de los niños que no presentan esta característica. Padres de familia, maestros docentes, científicos y terapeutas se esfuerzan en desarrollar sistemas ya sea neuropsicológicos, software lúdico y de otros tipos que auxilien a niños con TEA a que mejoren su atención para lograr aprender más rápido. De acuerdo a la experiencia de expertos del Centro de Atención Múltiple 51, un sistema mecánico que fuerce a utilizar sus dos hemisferios cerebrales, como lo permite un sistema de pedaleo y con un protocolo específico de tareas, les ayudará a concentrar más su atención y podrán aprender tareas básicas que les permita integrarse a escuelas convencionales. Las organizaciones educativas enfocadas en el tratamiento de niños con TEA son un número reducido y necesitan mejorar y desarrollar diferentes condiciones de aprendizaje para infantes con TEA para acelerar su integración en escuelas convencionales. Este artículo presenta la evolución de un sistema de pedaleo dinámico que se utiliza como complemento paralelo a la educación de un niño con TEA el cual consideramos que acelerará el desarrollo de sus habilidades y la posibilidad de integrarse más rápido a su entorno social.
\end{abstract}

Trastorno de Espectro Autista, Pedaleo dinámico, Diseño mecánico, Síndrome de Asperger

\begin{abstract}
The cognitive abilities of children with Autism Spectrum Disorder (ASD) are different from those of children who do not have this characteristic. Parents, teachers, scientists, and therapists strive to develop neuropsychological systems, ludic software, and others systems that help children with ASD improve their attention to learn faster. According to the experience of experts from the Multiple Attention Center 51, a mechanical system that forces them to use their two cerebral hemispheres, as allowed by a pedaling system and with a specific protocol of tasks, will help them to focus their attention allowing them to learn basic tasks that allow them to integrate into conventional schools. There are not many Educational organizations focused on treating children with ASD so they need to improve and develop different learning conditions for infants with ASD to accelerate their integration into conventional schools. This article presents the evolution of a dynamic pedaling system that is used as a parallel complement to the education of a child with ASD, which we believe will accelerate the development of their abilities and the possibility of integrating more quickly into their social environment.
\end{abstract}

Autism Spectrum Disorder, Dynamic Pedaling, Mechanical Design, Asperger's Syndrome

Citación: ORTIZ-SIMÓN, José Luis, AGUILERA-HERNÁNDEZ, Martha, MURILLO-RODRÍGUEZ, Miguel Randall y OLIVARES-CABALLERO, Daniel. Evolución y desarrollo de sistema interactivo para ayudar a infantes con Trastorno de Espectro Autista en su integración a escuelas convencionales. Revista de Educación Básica. 2020. 4-11:17-22.

\footnotetext{
*Correspondencia al Autor (Correo Electrónico: joseluisortizsimon@ hotmail.com)

$\dagger$ Investigador contribuyendo como primer autor.
} 


\section{Introducción}

Se estima que mundialmente uno de cada 160 niños tiene un TEA y es sabido que esta estimación puede ser menor a la realidad. Hay varios estudios que registran cifras considerablemente mayores. Los análisis que se han realizado en México indican que uno de cada 115 niños tiene esa condición. Cifras descritas de alrededor de 400,000 niños en el 2016 sugiere un problema urgente de salud pública en México. El TEA es un padecimiento no muy conocido y por lo tanto poco atendido ya que en México solo el $0.7 \%$ de las escuelas son Centros de Atención Múltiple [1].

Es actualmente considerado una problemática a nivel nacional. El TEA se caracteriza por un retraso o desviación en el desarrollo de las habilidades sociales, cognitivas y de comunicación en los niños, estas desviaciones tienen su inicio en los primeros años de vida [2].

Se caracteriza por problemas en las áreas cognitiva, social y de comunicación, y se acompaña de conductas estereotipadas entre ellas autoagresión, ecolalia y el apego estricto a rutinas. La educación y el apoyo comunitario son elementos fundamentales para el desarrollo de la comunicación y las competencias sociales, no sólo en niños con TEA [3].

Se han empleado una amplia variedad de modelos de intervención en las personas autistas, pero es pertinente señalar que no existe unanimidad en cuanto al método de intervención más idóneo, pues éste se debe adaptar al entorno y a las características individuales. Dada la gran heterogeneidad de los pacientes y a la dificultad en comparar las intervenciones, resulta complicado recomendar, en base a la evidencia científica, un método u otro. Se han llevado a cabo grandes avances en el tratamiento del TEA creando una propuesta pedagógica (COOPEDALEANDO: Ocio $\mathrm{y}$ actividad física para personas con TEA) para su auxilio sostenida en los siguientes pilares: Educación cognitiva, de autonomía personal, corporal, espiritual, emocional y social [4].
Los centros de atención múltiples y organizaciones educativas que se enfocan en el tratamiento de niños con TEA son un número reducido en comparación a los centros educativos convencionales (en México existen 207682 escuelas de las cuales el $0.7 \%$ son CAM). Por lo tanto, es necesario mejorar las condiciones de aprendizaje para, en consecuencia, mejorar las condiciones de vida y su integración natural en la sociedad. Es así que consideramos como un sistema interactivo de pedaleo dinámico en complemento paralelo a la educación de un niño con TEA acelerará el desarrollo de sus habilidades mejorando su entorno social y agilizando su integración a escuelas convencionales.

\section{Materiales y métodos}

Este proyecto resulto de la recomendación de un psicólogo experto del CAM51 en Nuevo Laredo Tamaulipas por lo que se decidió realizar el proyecto que colabore en el desarrollo y mejora de los centros anteriormente mencionados. Para el desarrollo del sistema de pedaleo se consideraron las características, efectos y niveles que conforman el Trastorno de Espectro Autista y verificar mediante la encuesta y la entrevista con el fin de diseñar por medio de dibujo mecánico una estructura que se adapte a las necesidades de los niños de manera amable y óptima, que en este caso fueron alumnos del Centro de Atención Múltiple \#51. Las varias visitas al CAM nos fueron retroalimentando de diversas problemáticas tanto mecánicas como sociales de la mano del equipo del Centro de Atención Múltiple formando con consejo capaz de responder inquietudes de terceros asegurando la seguridad y bienestar de los niños alentando el desarrollo de nuevo conocimiento a través de la investigación. Estructuralmente se tuvo que lograr un diseño ajustable y personalizable capaz de adaptarse a las necesidades físicas de quien lo usa dentro de un rango de ocho a doce años de edad buscando lograr objetivos generales a través de actividades específicas.

Las características de los niños estudiantes en el CAM51 son infantes de 5 a 10 años, con autismo, déficit de atención e hiperactividad. La estructura final presentada a continuación contiene las características y observaciones obtenidas del proyecto en base a su desarrollo por etapas. 
Estas etapas se distinguen por la selección de elementos, mejoras y observaciones respecto a su periodo de experimentación. Para la estructura final, se hizo un diseño preliminar utilizando elementos similares que cubriera con características basadas en los resultados de la investigación de campo previa.

Se inició el diseño contemplando las siguientes características básicas:

- Debe ser económico. El diseño debe tener partes de fácil adquisición y bajo costo.

- $\quad$ Debe ser seguro físicamente.

- Que tenga buen aspecto. Atractivo para los niños.

- Que tenga movimientos continuos. Velocidad media de pedaleo.

- Fácil de usar. De manejo simple y cómodo para manipular.

Fácil de manejar. Control de pedaleo sencillo.

Fácil de trasportar. Capacidad de transportarse fácilmente.

\section{Descripción de los sistemas}

Para cumplir con los requisitos iniciales de diseño y construcción se inició adaptando un sistema de pedaleo con un cuadro de bicicleta a una butaca estudiantil. Se cortó con la herramienta apropiada el cuadro de una bicicleta infantil y se fijó con soldadura a la butaca. Se acoplo un motor eléctrico a la cadena de tal forma que el pedaleo no fuera realizado por el infante, sino que fuera automático controlando la velocidad. Se realizo un control de velocidad a un motor de CD de automóvil y se probó con niños. Este modelo ofrece una butaca donde trabajar por el estudiante, sin embargo, las dimensiones no eran adecuadas para los niños del CAM. Además de que se dificultaba el pedaleo por la butaca y no podía recargarse el alumno. Era molesto para la espalda y se despegaban los pies del pedal. Desconcentraba mucho la actividad del niño en las tareas asignadas. Ver figura 1.

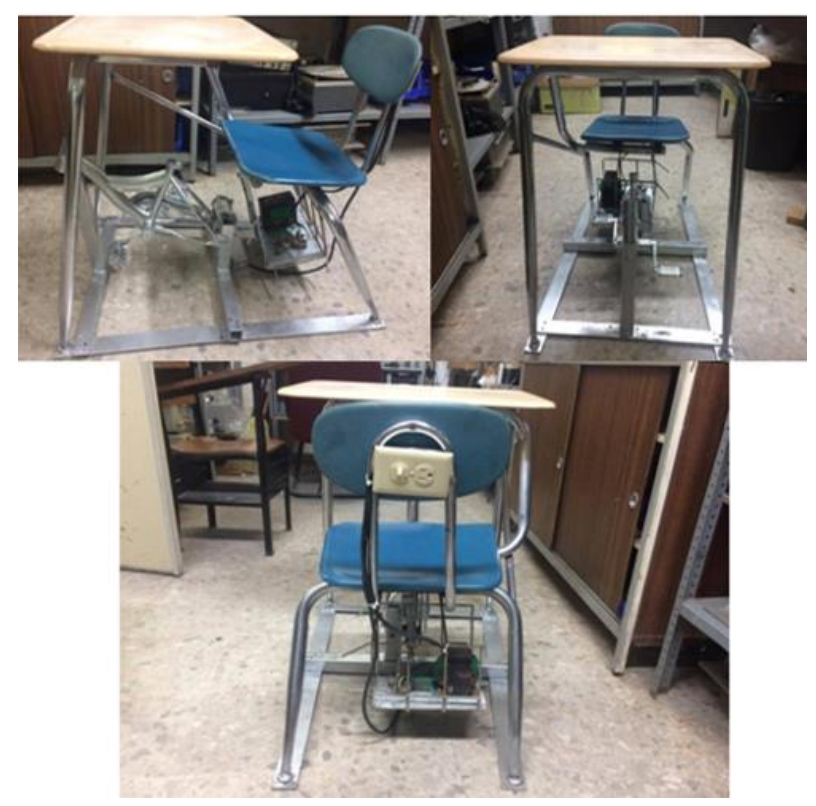

Figura 1 Primer diseño con butaca y cuadro de bicicleta

El segundo modelo se adaptó un sistema de pedaleo usado para ejercitarse, con ajustes de velocidad y con posibilidad de programar secuencias de giro, cambios de velocidad y tiempo. De igual forma se acoplo a una butaca estudiantil. Al momento de probarla con niños, se tuvo resultados no convenientes ya que, a pesar de que se tenía más control de la velocidad, se separaban los pies de los pedales y perdían la atención a la tarea asignada. No hubo mejoras significativas (Figura 2).

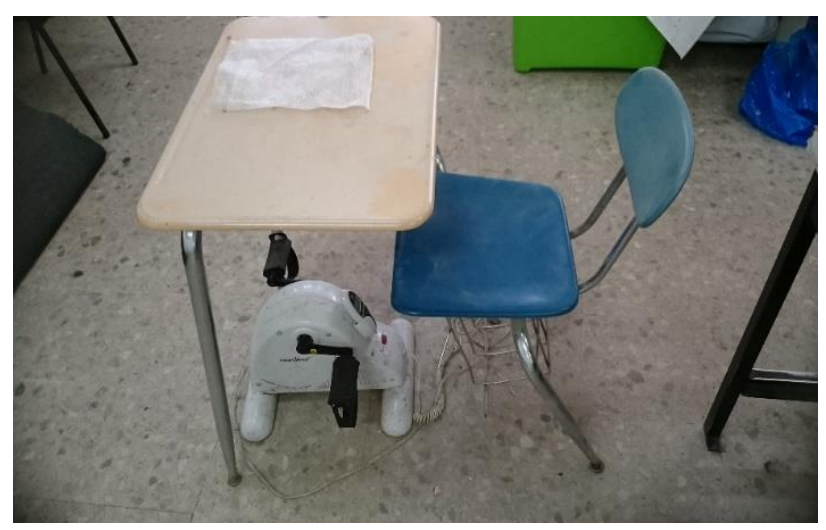

Figura 2 Diseño del sistema con sistema de pedaleo con control de velocidad

Posteriormente tomamos medidas de los niños, esto altura y longitud de piernas. De una muestra de 23 alumnos entre 6 y 9 años, promediaron una altura de $1.17 \mathrm{~m}$ y longitud de entrepierna de $0.64 \mathrm{~m}$. En base a esas dimensiones se diseñó una estructura donde se le adapto el sistema de pedaleo y un asiento para que descansara el niño. 
$\mathrm{Y}$ puesto que ellos utilizan mesas especiales para cada tarea que se les asigna, ahora debía de cumplir con el hecho de que pudiera acomodarse a la mesa de trabajo que se les asignara. Sin embargo, después de varias pruebas realizadas, notamos que debíamos fijar de algún modo los pedales a los pies de los niños y poder ajustar al menos con una variación de $5 \mathrm{~cm}$ la altura y de igual forma la distancia del asiento sobre los pedales. El diseño que se obtuvo se muestra en la figura 3.

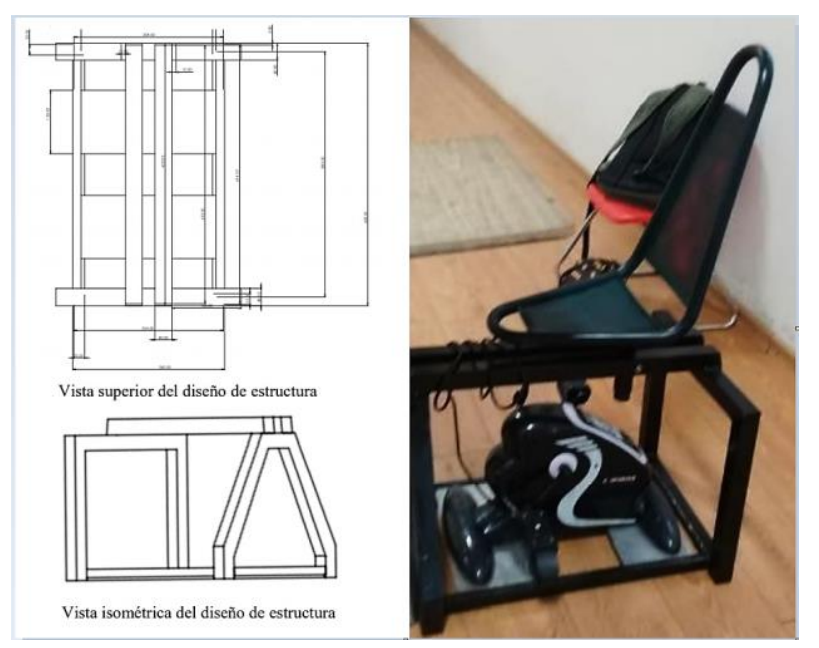

Figura 3 Diseño con estructura de acuerdo con medidas promedio de los niños

Finalmente llegamos a un diseño el cual se le acoplaron unas sandalias comerciales donde entra el pie del niño sin quitar sus zapatos y se fija con correa. Se rediseño el asiento más ergonómico a la anatomía infantil y permitiendo un ajuste de altura de hasta $6 \mathrm{~cm}$ y $12 \mathrm{~cm}$ para desplazar el asiento hacia atrás o adelante. Se modificó la estructura a un diseño menos pesado de manera que las maestras lo puedan fácilmente acoplar a cualquier área de trabajo y con menos estructura metálica para permitir movimientos más seguros y firmes.

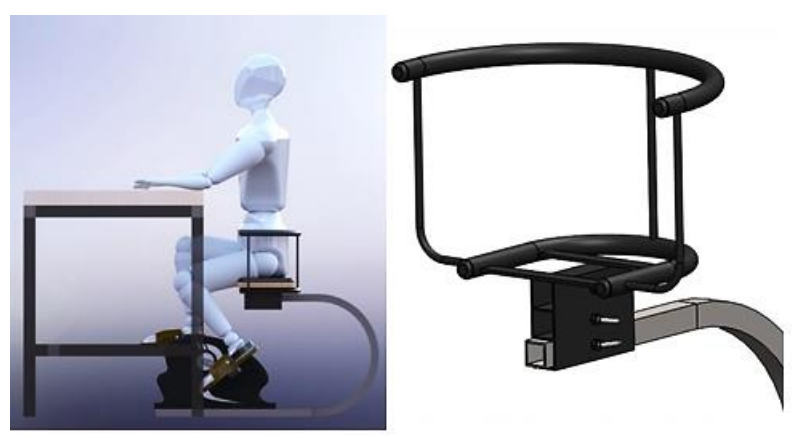

Figura 4 Diseño con sandalias y estructura más ligera con ajustes
Por otro lado, la estructura de este rediseño es ligero utilizando menos piezas comparado con el diseño anterior. Esto ayuda a que las maestras, o quienes ayudarán al niño, a poder trasladar el sistema de manera segura y con menor esfuerzo. El diseño actual se utiliza tubo PTR de $1 \frac{1}{2}$ así reduciendo significativamente el peso del sistema. Por otra parte, se hizo consideración en el uso del aparato, ya que el control se encuentra en la parte trasera, así que se tomó la idea de poner un tubo curvo, para fácil acceso y visibilidad de las velocidades en la que va operar y que no ocupe mucho espacio. El asiento se encuentra más cerca al sistema de pedaleo con la finalidad de no perder movilidad al momento de que la pierna del niño forme 90 grados respecto a la cadera. Se mejoró el espacio de trabajo del pie y la postura del niño facilitándole recargar sus codos sobre la mesa de trabajo (Figura 5).

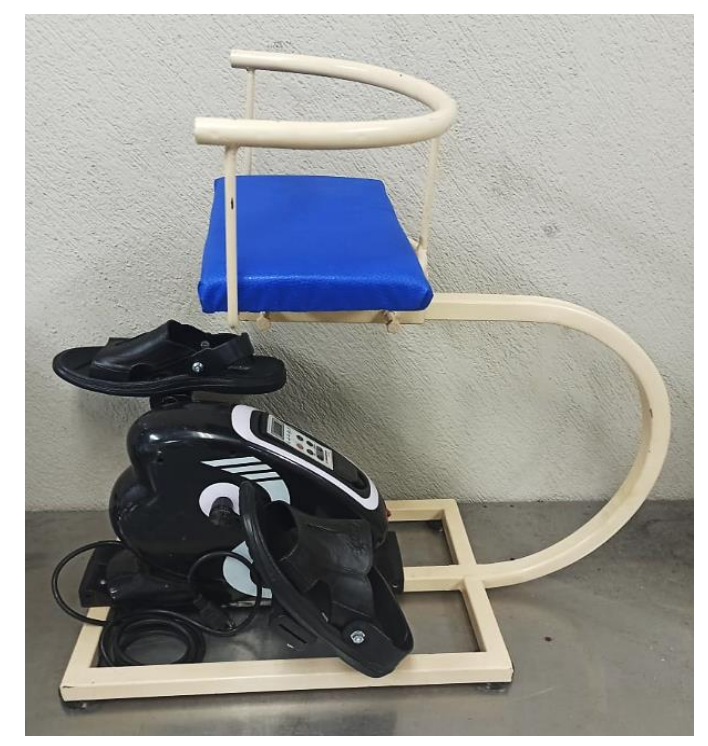

Figura 5 Estructura ergonómica y con sandalias

\section{Resultados}

En la visita de interacción del sistema de pedaleo dinámico con los niños del CAM se tomaron imágenes y se optó por reducir velocidad al motor para que todos los niños siguieran una velocidad estándar al momento del encendido del pedaleo. Cabe mencionar que a pesar de ser niños con rasgos de hiperactividad no se perdía objetividad de su parte para hacer uso del sistema, característica que fue útil para valorar alcances a corto plazo. Por medio de pruebas determinadas por las maestras y expertas del CAM51 se pretendió medir la atención, concentración y permanencia del alumno en el sistema de pedaleo dinámico. Se aplicó una prueba de uso a 26 alumnos (Figura 6).

MUÑOZ-LOPEZ, Temístocles, MARTÍNEZ-SOSA, Vanessa, CEPEDA-GONZÁLEZ, Cristina y CERVANTES-MARMOLEJO, Claudia. El Laboratorio Nómada del Agua, Con-Ciencia ambiental desde la Educación Básica. Revista de Educación Básica. 2018 


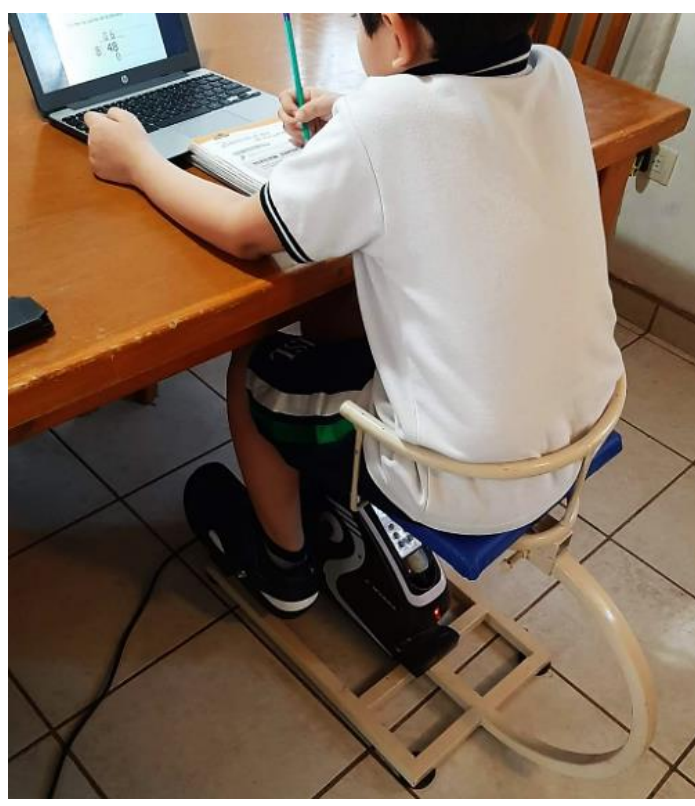

Figura 6 Prueba de sistema en alumno

A solo dos de ellos se les hizo un ajuste del asiento que se logró en menos de un minuto, con una llave perica. Hemos notado que, para niños de 6 a 10 años, con quienes se probó el sistema, podrán realizar las tareas asignadas por las maestras del CAM51. El peso del sistema no sobrepasa los $15 \mathrm{~kg}$, y las dimensiones menores a $1 \mathrm{~m}^{2}$ permiten acomodarse en las mesas de trabajo del CAM. La programación del sistema es muy amigable y el requerimiento eléctrico es el convencional.

\section{Conclusiones}

El sistema de pedaleo dinámico presenta una opción experimental para las escuelas que desean innovar sus estrategias de enseñanza, sin embargo, nos enfrentamos a un proceso en el que estamos obligados a generar información formalizada y aceptada por un consejo ético que nos permita su implementación de manera segura tanto para los sujetos como para sus responsables. Esta formalización del conocimiento también depende de la constante confirmación de resultados, que, aunque se tienen algunos avances, pueden ser más específicos y precisos en cuestión de objetivos realizados en determinados periodos de tiempo evaluados con herramientas de estadística compleja, y tomando referencias de instituciones de primer mundo brindando un apoyo fidedigno a los jóvenes que padecen TEA.
Los programas de estudios de escuelas de educación especial plantean objetivos generales que no permiten el desarrollo de habilidades particulares. Por lo tanto, se propone una serie de actividades desglosadas a partir de los objetivos de los programas de estudio basados principalmente en los rubros de lenguaje y comunicación y pensamiento matemático. Creemos que este sistema mecánico de pedaleo interactivo, ayudará a generar los datos necesarios para demostrar que se puede lograr concentrar la atención de los niños en tareas específicas para que el aprendizaje de parte de los niños del CAM sea más eficiente y logren una integración a escuelas convencionales con mayor rapidez.

\section{Referencias}

[1] INEGI. (2014). Censo de Escuelas, Maestros y Alumnos de Educación Básica y Especial. 11 Nov 2020, de INEGI Sitio web:

https://www.uv.mx/personal/kvalencia/fil es/2013/09/INEGI-2014-Censo-

Escolar.pdf

[2] Felix Ambrosio Gallardo. (6 Nov 2007). Trastornos del espectro autista. Revista Mexicana de Pediatría, 74, 269-276.

[3] F Volkmar 1, E H Cook Jr, J Pomeroy, G Realmuto, P Tanguay. (Dec 1999). Practice parameters for the assessment and treatment of children, adolescents, and adults with autism and other pervasive developmental disorders. American Academy of Child and Adolescent Psychiatry Working Group on Quality Issues. J Am Acad Child Adolesc Psychiatry, 38, 32-54.

[4] Diario Oficial de la Federación. (2016). NORMA Oficial Mexicana NOM-012SSA3-2012. 11-12-2020, de Secretaría de Gobernación Sitio web: http://dof.gob.mx/nota_detalle.php?codig $\mathrm{o}=5284148 \&$ fecha $=04 / 01 / 2013$

[5] Carpenter, M., Pennington, B. F., \& Rogers, S. J. (2002). Interrelations among socialcognitive skills in young children with autism. Journal of Autism and Developmental Disorders, 32, 91-106. 
[6] Leekam, S., Hunnisett, E., \& Moore, C. (1998). Targets and cues: Gaze following in children with autism. Journal of Child Psychology and Psychiatry, 39, 951-962.

[7] Leekam, S., Lopez, B., \& Moore, C. (2000). Attention and joint attention in preschool children 